\title{
Levels of microRNA-181b and plasminogen activator inhibitor-1 are associated with hypertensive disorders complicating pregnancy
}

\author{
YAN-SHAN CHEN ${ }^{1}$, LING SHEN ${ }^{1}$, RUI-QIN MAI ${ }^{2}$ and YING WANG ${ }^{1}$ \\ Departments of ${ }^{1}$ Gynecology and Obstetrics and ${ }^{2}$ Clinical Laboratory, \\ The First Affiliated Hospital of Shantou University Medical College, \\ Shantou, Guangdong 515041, P.R. China
}

Received February 19, 2014; Accepted August 7, 2014

DOI: $10.3892 /$ etm.2014.1946

\begin{abstract}
The aim of the present study was to explore the association between the expression of microRNA (miRNA)-181b and plasminogen activator inhibitor-1 (PAI-1) in the placental tissue of pregnant females with a hypertensive disorder complicating pregnancy (HDCP). Placental tissue samples were obtained from 48 patients with HDCP and 40 females with a normal pregnancy. The levels of miRNA-181b and PAI-1 mRNA were determined by the reverse transcription-quantitative polymerase chain reaction (RT-qPCR). The expression of PAI-1 protein was analyzed by western blotting. Vascular smooth muscle cells (VSMCs) were transfected with the pEGP-miRNA-181b plasmid using Lipofectamine ${ }^{\circledR} 2000$. Transfection efficiency was confirmed by immunohistochemical analysis. The levels of miRNA-181b in the placental tissue of patients with HDCP were lower than those in the control group, whereas the levels of PAI-1 mRNA in the placental tissue of patients with HDCP were higher than those in the control group. The expression of the PAI-1 protein in the HDCP group was higher than that in the control group. Following transfection of VSMCs with plasmid pGCMV/EGFP/miRNA-181b, the levels of PAI-1 mRNA were reduced while the levels of miRNA-181 were upregulated. Furthermore, the expression levels of PAI-1 protein were lower than those in the control group. The levels of miRNA-181b and PAI-1 mRNA were strongly associated with HDCP. Thus, miRNA-181b may play an important role in the regulation of PAI-1. PAI-1 and miRNA-181b may be novel biomarkers to be used in HDCP therapy.
\end{abstract}

Correspondence to: Professor Ying Wang, Department of Gynecology and Obstetrics, The First Affiliated Hospital of Shantou University Medical College, 57 Changping Road, Shantou, Guangdong 515041, P.R. China

E-mail: xqyy166@163.com

Key words: hypertensive disorders complicating pregnancy, microRNA-181b, plasminogen activator inhibitor-1

\section{Introduction}

Hypertensive disorder complicating pregnancy (HDCP) is a common clinical obstetric complication with an incidence rate of 5\% in China (1). Due to a lack of effective means of prevention and treatment, HDCP threatens the lives of pregnant females and their fetuses. The clinical manifestations of HDCP are mainly hypertension, proteinuria and edema, as well as convulsion, coma and heart or kidney failure when aggravated (1). Studies on molecular biomarkers for the early stages of HDCP development are important for the prevention and treatment of HDCP. microRNA (miRNA)-181b, a member of the miRNA-181 family, has been revealed to be involved in the proliferation, invasion and apoptosis of tumor cells that are associated with tumor invasiveness $(2,3)$. Plasminogen activator inhibitor-1 (PAI-1), a novel target for the clinical treatment of cardiovascular disease (4), inhibits fibrinolysis, induces barriers for extracellular matrix degradation, impacts the invasiveness of cells (5) and plays an important role in vascular structural and functional changes (6).

Previous studies on the pathological mechanisms of HDCP have demonstrated that the placenta plays an important role in the occurrence and development of HDCP; the mechanism is associated with the reduced invasiveness of trophoblastic cells and pathological changes in small branches of the main uterine artery $(7,8)$. Furthermore, it is hypothesized that the shallow invasion of trophoblastic cells into the uterus, dysfunctional physiological recovery of the spiral arterioles and placental ischemia and hypoxia are associated with HDCP (9). Studies have indicated that miRNA-181 has an effect on the proliferation of cells, the regulation of angiogenesis, the conditioning of the immune system (10), tumor invasion and apoptosis (11). Furthermore, miRNA-181 has been shown to exhibit clinical value by regulating $\mathrm{Bcl}-2$, OPN and K-ras genes $(12,13)$. Notably, miRNA-181b has been demonstrated to promote the proliferation and invasiveness of multiple tumor cells and is closely associated with tumor apoptosis (14). In addition, a previous study revealed that miRNA-181a promotes the invasiveness of trophoblastic cells and is highly expressed in the placental tissue of patients with HDCP (15). However, the effect of miRNA-181b on the occurrence and development of HDCP has not, to the best of our knowledge, been previously studied. 
PAI-1 is mainly expressed in vascular endothelial cells and vascular smooth muscle cells (VSMCs), and plays an important role in the hypertrophy, proliferation and remodeling of the VSMCs (16). Bioinformatic studies have indicated that the 3'-untranslated region of PAI-1 mRNA is complementary to miRNA-181b $(17,18)$. Therefore, we hypothesized that miRNA-181b may be capable of regulating the expression of PAI-1, which affects vascular structural remodeling and function, thereby promoting the occurrence and development of HDCP. In the present study, the mechanisms of action of miRNA-181b and PAI-1 in the occurrence and development of HDCP were investigated.

\section{Materials and methods}

Subjects. Placental tissue was collected from 48 puerperae with HDCP (HDCP group) and 40 puerperae with normal deliveries (normal control group) between October 2011 and October 2013. In the HDCP group, 28 females had gestational hypertension (high blood pressure following 20 weeks of pregnancy; no proteinuria), 15 females had mild pre-eclampsia (systolic pressure $\geq 140 \mathrm{mmHg}$ or diastolic pressure $\geq 90 \mathrm{mmHg}$; $24 \mathrm{~h}$ proteinuria $\geq 300 \mathrm{mg}$ ) and five females had severe pre-eclampsia (systolic pressure $\geq 160 \mathrm{mmHg}$ or diastolic pressure $\geq 100 \mathrm{mmHg} ; 24 \mathrm{~h}$ proteinuria $\geq 2 \mathrm{~g}$ ). The ages of the females in the HDCP group ranged between 24 and 34 years, with an average age of 28.5 years and a median age of 27 years. The ages of the females in the normal control group ranged between 25 and 30 years, with an average age of 26.5 years and a median age of 25 years. Written informed consent was obtained from all participants and all experiments were approved by the Ethics Committee of Shantou University (Shantou, China).

Reagents. VSMCs were purchased from the Cell Bank of Chinese Academy of Sciences (Shanghai, China). Plasmids for pGCMV/EGFP/miRNA-181b and the negative control were constructed by Shanghai GenePharma Co., Ltd. (Shanghai, China). Rabbit anti-human PAI-1 polyclonal antibody was purchased from Abcam (Cambridge, MA, USA). An RNA extraction kit (spin column) was purchased from Sangon Biotech Co., Ltd. (Shanghai, China). A reverse transcription kit was purchased from Chengdu Boruike Biotech Co., Ltd. (Chengdu, China). First-strand miRNA/cDNA synthesis kits were purchased from Beijing CoWin Biotech Co., Ltd. (Beijing, China). SYBR Green reverse transcription-quantitative polymerase chain reaction (RT-qPCR) reagents were purchased from Kapa Biosystems (Boston, MA, USA). Lipofectamine $^{\circledR} 2000$ was purchased from Invitrogen Life Technologies (Carlsbad, CA, USA).

Total RNA extraction. Total RNA from the placental tissue and VSMCs was extracted using the total RNA extraction kit in accordance with the manufacturer's instructions (Sangon Biotech Co., Ltd.). The quality of the extracted RNA was confirmed by RNA molecular electrophoresis and a NanoDrop ND-1000 UV-Vis spectrophotometer (NanoDrop Technologies, Inc., Wilmington, DE, USA) at an optical density ratio of 260/280 nm.

$R T$. Total RNA (1 $\mu \mathrm{g})$ was used for RT. RNA template (5 $\mu \mathrm{l})$, Escherichia coli poly(A) polymerase $(0.4 \mu \mathrm{l})$, poly(A) polymerase B buffer $(2 \mu 1)$, adenosine triphosphate solution (2 $\mu \mathrm{l})$ and RNase-free double distilled water (10.6 $\mu \mathrm{l})$ were added into a pre-cooled RNase-free Eppendorf tube and incubated at $37^{\circ} \mathrm{C}$ for $60 \mathrm{~min}$ prior to poly(A) modification. The mixture $(4 \mu \mathrm{l})$ was subsequently added to the $\mathrm{RT}$ reaction mixture $(20 \mu \mathrm{l})$ containing RT primer $(2 \mu \mathrm{l})$, RT buffer $(4 \mu \mathrm{l})$, deoxynucleotide triphosphate mix $(1 \mu \mathrm{l})$, TUREscriptH-RTase $(0.9 \mu \mathrm{l})$ and $\mathrm{RNase}$-free $\mathrm{H}_{2} \mathrm{O}(8.9 \mu \mathrm{l})$. The $\mathrm{RT}$ reaction was maintained at $42^{\circ} \mathrm{C}$ for $50 \mathrm{~min}$ and heated to $70^{\circ} \mathrm{C}$ for $15 \mathrm{~min}$ prior to termination. The cDNA was stored at $-20^{\circ} \mathrm{C}$.

Western blot analysis. Placental tissue (100 mg) was obtained from individuals in the HDCP and normal control groups and ground into a powder in liquid nitrogen. Radioimmunoprecipitation assay lysis buffer and protease inhibitors were added with thorough mixing prior to incubation at $4^{\circ} \mathrm{C}$ overnight. The following day, the samples were centrifuged at $15,680 \mathrm{x}$ g at $4^{\circ} \mathrm{C}$ for $10 \mathrm{~min}$ and the supernatants were stored in aliquots. Prior to electrophoresis, sample loading buffer (2X) was mixed thoroughly with an aliquot of the sample before denaturation for $5 \mathrm{~min}$ in a boiling water bath. The mixture $(10 \mu \mathrm{l})$ was loaded onto the gel for sodium dodecyl sulfate polyacrylamide gel electrophoresis at a constant $80 \mathrm{~V}$ and electrically transferred onto a polyvinylidene difluoride membrane at constant $200 \mathrm{~mA}$ in an ice bath for $2 \mathrm{~h}$. The membrane was blocked by skimmed milk $(50 \mathrm{~g} / \mathrm{l})$ for $1 \mathrm{~h}$ at room temperature. Primary antibodies against PAI-1 (1:1,000; Abcam) and $\beta$-actin (1:5,000; Abcam) were added prior to incubation with agitation overnight at $4^{\circ} \mathrm{C}$. Following rinsing with phosphate-buffered saline (PBS) with Tween 20 three times for $10 \mathrm{~min}$, secondary antibodies labeled with horseradish peroxidase (goat anti-mouse, 1:5,000; goat anti-rabbit, 1:2,000; Abcam) were added prior to incubation at room temperature for $1 \mathrm{~h}$. This was followed by rinsing with PBS with Tween 20 three times for $10 \mathrm{~min}$. The immunoreactive bands were visualized by electrochemiluminescence.

$R T-q P C R$. U6 and GAPDH were used as internal controls for the quantification of miRNA-181b and PAI-1, respectively. The miRNA-181b reaction system was composed of the RT-qPCR mix $(10 \mu 1)$, upstream and downstream primers (0.5 $\mu$ l each; Invitrogen Life Technologies), cDNA (5 $\mu 1)$ and double distilled $\mathrm{H}_{2} \mathrm{O}(13 \mu \mathrm{l})$. The amplification conditions were as follows: Initial denaturation at $95^{\circ} \mathrm{C}$ for $10 \mathrm{~min}$, denaturation at $95^{\circ} \mathrm{C}$ for $30 \mathrm{sec}$, annealing at $65^{\circ} \mathrm{C}$ for $30 \mathrm{sec}$ and extension at $72^{\circ} \mathrm{C}$ for $1 \mathrm{~min}$, repeated for 40 cycles. The samples were analyzed in triplicate. The PAI-1 reaction system was composed of the RT-qPCR mix (10 $\mu 1)$, upstream and downstream primers (0.5 $\mu 1$ each; Invitrogen Life Technologies), cDNA $(1 \mu \mathrm{l})$ and double distilled $\mathrm{H}_{2} \mathrm{O}(8 \mu \mathrm{l})$. The amplification conditions were as follows: Initial denaturation at $95^{\circ} \mathrm{C}$ for $10 \mathrm{~min}$, denaturation at $95^{\circ} \mathrm{C}$ for $1 \mathrm{~min}$, annealing at $58^{\circ} \mathrm{C}$ and $72^{\circ} \mathrm{C}$ for $30 \mathrm{sec}$, and extension at $72^{\circ} \mathrm{C}$ for $1 \mathrm{~min}$, repeated for 40 cycles. The samples were analyzed in triplicate.

Cell transfection. On the day prior to transfection, VSMCs $\left(1 \times 10^{5}\right)$ in the logarithmic phase were seeded onto 24-well plates and divided into the normal control, miRNA-181b-VSMC and miRNA negative control groups. The cells were cultured in antibiotic-free, high-glucose Dulbecco's modified Eagle's 
medium (DMEM) and 10\% fetal bovine serum (FBS). The cells were transfected when $70 \%$ confluency was reached. The pGCMV/EGFP/miRNA-181b plasmid $(2 \mu \mathrm{g})$ and Lipofectamine $2000(1 \mu \mathrm{l})$ were mixed individually with Opti-MEM ${ }^{\circledast} \mathrm{I}(50 \mu 1$; Invitrogen Life Technologies) in separate Eppendorf tubes and left to stand for $5 \mathrm{~min}$ prior to being mixed into one tube. After standing for $20 \mathrm{~min}$ at room temperature, the mixture was added into the wells of the culture plates. After $6 \mathrm{~h}$, the medium was replaced with fresh high-glucose DMEM and 10\% FBS. After 48 h, green fluorescence was observed under an inverted fluorescence microscope (X51; Olympus Corporation, Tokyo, Japan) for the preliminary analysis of transfection efficiency. After 48 and $72 \mathrm{~h}$ of transfection, the cells were analyzed to determine the expression levels of the genes and proteins.

Statistical analysis. The results were analyzed using SPSS 16.0 software (SPSS, Inc., Chicago, IL, USA). All results are expressed as the mean \pm standard deviation. Two groups of mean values were compared using the Student's t-test. $P<0.05$ was considered to indicate a statistically significant difference.

\section{Results}

$H D C P$ reduces the levels of miRNA-181b but increases the levels of PAI-1 mRNA in placental tissue. To determine the levels of miRNA-181b and PAI-1 mRNA in the placental tissue from pregnant females in the HDCP and control groups, RT-qPCR was performed. The results revealed that the level of miRNA-181b in the placental tissue from the HDCP group was significantly lower than that in the placental tissue from the control group $(\mathrm{P}<0.05$; Fig. 1A). By contrast, the level of PAI-1 mRNA in the placental tissue from the HDCP group was higher than that in the placental tissue from the control group. The levels of PAI-1 mRNA in the placental tissue from patients with gestational hypertension, mild pre-eclampsia and severe pre-eclampsia increased 1.98-, 2.79- and 5.8-fold, respectively, compared with those from the control group (Fig. 1B). These data indicated that HDCP reduced the levels of miRNA-181b but increased the levels of PAI-1 mRNA in placental tissue.

HDCP increases the protein expression of PAI-1. To measure PAI-1 protein expression, western blot analysis was performed. Western blotting revealed that the expression of PAI-1 protein in placental tissue from the HDCP group was significantly higher than that in placental tissue from the normal control group $(\mathrm{P}<0.05$; Fig. 2). This result suggested that HDCP increased the protein expression of PAI-1.

Transfection of VSMCs with the $p G C M V / E G F P / m i R N A-181 b$ plasmid enhances the levels of miRNA-181b and reduces the levels of PAI-1 mRNA in VSMCs. To investigate the expression levels of miRNA-181b and PAI-1 in the VSMCs, the pGCMV/EGFP/miRNA-181b plasmid was transfected into the VSMCs and the expression of miRNA-181b was detected using RT-qPCR. Microscopic observation following 48 h (Fig. 3) of transfection revealed that the fluorescence in the transfected VSMCs was distributed evenly (Fig. 3B), with a wider range and a higher density than that in the cells of the negative
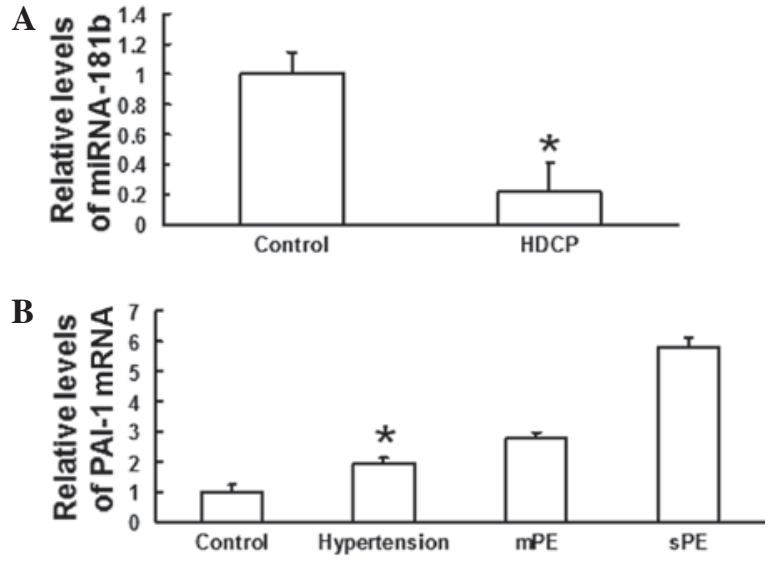

Figure 1. Levels of (A) miRNA-181b and (B) PAI-1 mRNA in the normal control and HDCP groups. The HDCP group was divided into three subgroups of hypertension, $\mathrm{mPE}$ and $\mathrm{SPE}$. Data were normalized against those of the normal control group and expressed as the mean \pm standard deviation. $\mathrm{P}<0.05$ was considered significantly different. *Significant difference from the control. miRNA-181b, microRNA-181b; PAI-1, plasminogen activator inhibitor-1; HDCP, hypertensive disorder complicating pregnancy; mPE, mild pre-eclampsia; sPE, severe pre-eclampsia.

A

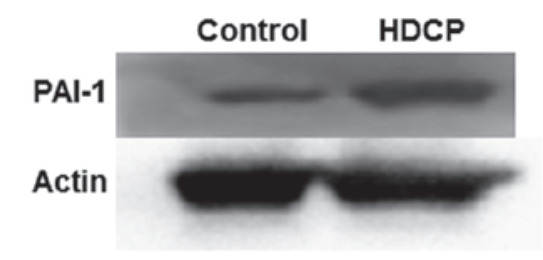

B

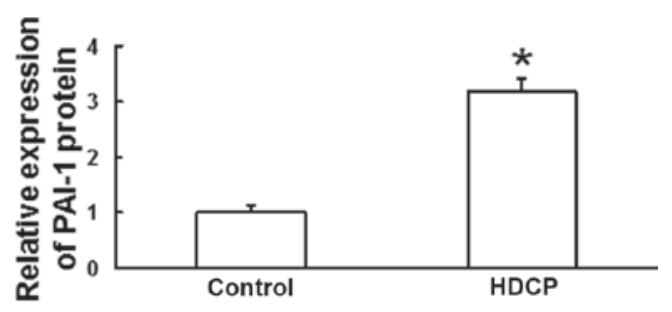

Figure 2. Expression of PAI-1 protein in placental tissue. (A) Western blot analysis of PAI-1 protein expression in the normal control and HDCP groups. (B) Quantification of the relative expression of PAI-1 protein in the normal control and HDCP groups. Data from the HDCP group were normalized against those from the normal control group and expressed as the mean \pm standard deviation. $\mathrm{P}<0.05$ was considered significantly different. *Significant difference from the control. PAI-1, plasminogen activator inhibitor-1; HDCP, hypertensive disorder complicating pregnancy.

control group (Fig. 3A). Analysis by RT-qPCR demonstrated that the level of miRNA-181b in the VSMCs following $48 \mathrm{~h}$ of transfection was significantly higher than that in normal VSMCs (P<0.05; Fig. 3C). Furthermore, the levels of PAI-1 mRNA in the VSMCs following $48 \mathrm{~h}$ of transfection were significantly lower than those in normal VSMCs $(\mathrm{P}<0.05$; Fig. 3D). These data demonstrated that transfection of VSMCs with the pGCMV/EGFP/miRNA-181b plasmid enhanced the levels of miRNA-181b in the VSMCs, in which the levels of PAI-1 mRNA were reduced.

Transfection of VSMCs with the $p G C M V / E G F P / m i R N A-181 b$ plasmid reduces PAI-1 protein expression. To determine PAI-1 protein expression in VSMCs transfected with the 
A

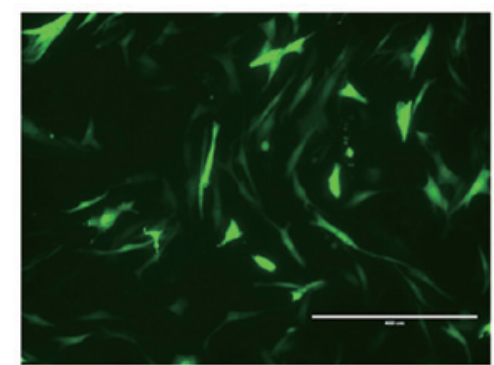

C

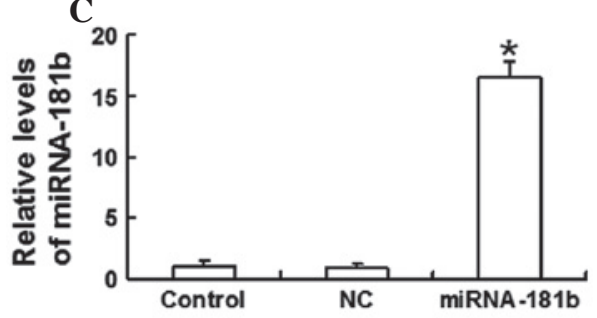

B

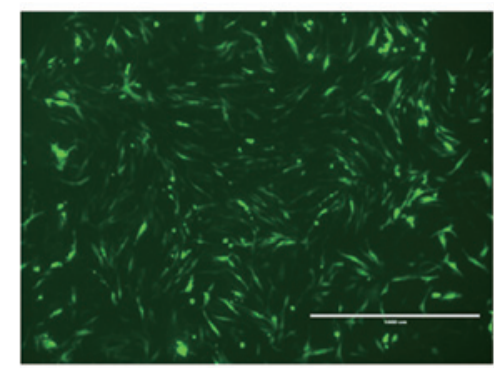

D

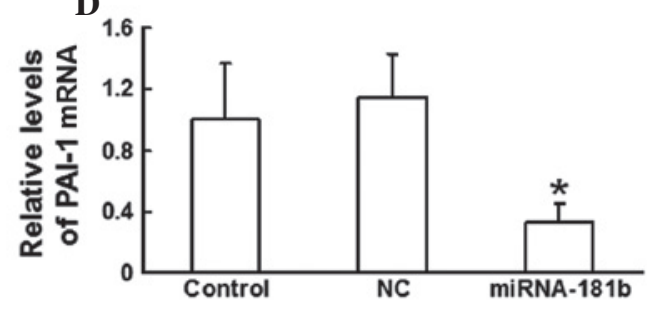

Figure 3. Fluorescence visualization of the VSMCs transfected with (A) an empty vector and (B) miRNA-181b plasmid. (C and D) Levels of (C) miRNA-181b and (D) PAI-1 mRNA in the normal control, NC and miRNA-181b groups following $48 \mathrm{~h}$ of transfection, as determined by reverse transcription-quantitative polymerase chain reaction. Data were normalized against those of the normal control group and expressed as the mean \pm standard deviation. ${ }^{*} \mathrm{P}<0.05$, vs. normal control. VSMC, vascular smooth muscle cell; miRNA-181b, microRNA-181b; PAI-1, plasminogen activator inhibitor-1; NC, negative control.
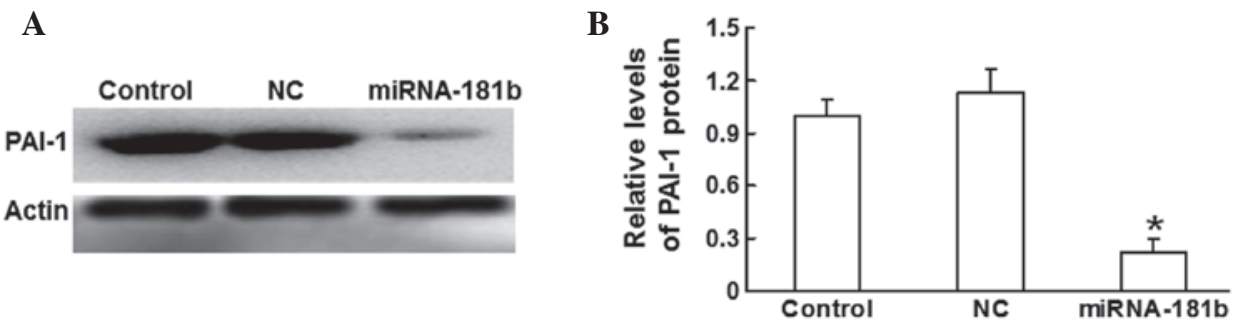

Figure 4. Expression levels of PAI-1 protein in VSMCs. (A) Western blot analysis of PAI-1 protein expression in the normal control, NC and miRNA-181b (miRNA-181b-VSMC) groups. (B) Quantification of the relative expression of PAI-1 protein in the normal control, NC and miRNA-181b groups. Data were normalized against those of the normal control group and expressed as the mean \pm standard deviation. " $\mathrm{P}<0.05$, vs. normal control. VSMC, vascular smooth muscle cell; miRNA-181b, microRNA-181b; PAI-1, plasminogen activator inhibitor-1; NC, negative control.

pGCMV/EGFP/miRNA-181b plasmid, western blotting was used to analyze the extracted proteins from VSMCs following $72 \mathrm{~h}$ of transfection. The results revealed that the protein expression of PAI-1 in VSMCs transfected with pGCMV/EGFP/miRNA-181b was significantly lower than that in the normal control and negative control groups $(\mathrm{P}<0.05$; Fig. 4). These data indicated that transfection of VSMCs with the $\mathrm{pGCMV/EGFP/miRNA-181b}$ plasmid reduced PAI-1 protein expression.

\section{Discussion}

In the present study, the levels of miRNA-181b in the placental tissue from the 48 patients with HDCP were lower than those in the placental tissue of normal pregnant females. However, the mRNA expression levels of PAI-1 in the placental tissue from patients with HDCP were higher compared with those in the placental tissue of normal pregnant females. In addition, the mRNA expression levels of PAI-1 gradually increased with the development of HDCP at different clinical stages (hypertension, mild pre-eclampsia and severe pre-eclampsia). By contrast, the levels of miRNA-181b were independent of the clinical stages of HDCP (data not shown).
Western blot analysis revealed that the protein expression of PAI-1 in the HDCP group was significantly enhanced compared with that in the control group, indicating that PAI-1 plays an important role in HDCP. By contrast, in hyperglycemia, PAI-1 protein expression has been found to decrease (19). This inconsistency indicates that PAI-1 protein expression may be regulated differently between hyperglycemia and HDCP. The changes in the levels of miRNA-181b in the placental tissue were contrary to the changes in the levels of PAI-1, suggesting that miRNA-181b may be involved in the regulation of PAI-1 expression.

Previous studies have demonstrated that miRNA-181 may regulate embryo implantation, placentation and decidualization via the regulation of the focal adhesion signaling pathway $(20,21)$. These observations indicate that the miR-181 family may play an important role in the development of the embryo and placenta. Therefore, to further investigate the role of miRNA-181b, an miRNA-181b eukaryotic expression model was constructed by transfecting VSMCs with miRNA-181b. The RT-qPCR results revealed that the levels of miRNA-181b increased by 16.5 -fold, whereas the mRNA expression levels of PAI-1 were reduced by $67 \%$ of the levels prior to transfection. Furthermore, the protein expression level of PAI-1 decreased to $22 \%$ of the level prior 
to transfection. These results demonstrated that PAI-1 expression was reduced when miRNA-181b expression was elevated, indicating that miRNA-181b may be involved in the regulation of PAI-1 expression. However, chromatin immunoprecipitation sequencing is required prior to confirming that PAI- 1 is the target gene for miRNA-181b regulation.

In conclusion, miRNA-181b and PAI-1 were shown to be pathologically expressed in the placental tissue of patients with HDCP. Therefore, miRNA-181b plays an important role in the occurrence and development of HDCP, possibly through participating in the regulation of PAI-1, although the precise underling mechanism requires further investigation. Thus, miRNA-181b and PAI-1 have a potential clinical value in the early diagnosis and prevention of HDCP.

\section{Acknowledgements}

This study was supported by The First Affiliated Hospital of Shantou University Medical College. The authors thank Dr Guohong Zhang for the invaluable instruction.

\section{References}

1. Zhou J, Xiao XM and Wu YH: Expression of interferon- $\gamma$ in decidual natural killer cells from women with hypertensive disorder complicating pregnancy. J Obstet Gynaecol Res 40: 670-676, 2014.

2. Yang L, Wang YL, Liu S, et al: miR-181b promotes cell proliferation and reduces apoptosis by repressing the expression of adenylyl cyclase 9 (AC9) in cervical cancer cells. FEBS Lett 588: 124-130, 2014

3. Shi L, Cheng Z, Zhang J, et al: hsa-mir-181a and hsa-mir-181b function as tumor suppressors in human glioma cells. Brain Res 1236: 185-193, 2008.

4. Simone TM, Archambeault J and Higgins PJ: Small molecule targeting of PAI-1 function: a new therapeutic approach for treatment of vascular stenosis. J Mol Genet Med 7: 1000059, 2013

5. Gomes-Giacoia E, Miyake M, Goodison S and Rosser CJ: Targeting plasminogen activator inhibitor-1 inhibits angiogenesis and tumor growth in a human cancer xenograft model. Mol Cancer Ther 12: 2697-2708, 2013.

6. Boe AE, Eren M, Murphy SB, et al: Plasminogen activator inhibitor-1 antagonist TM5441 attenuates N $\omega$-nitro-L-arginine methyl ester-induced hypertension and vascular senescence. Circulation 128: 2318-2324, 2013.
7. Li LX, Liu YL, Wen JG, et al: Expression and significance of aquaporin 1 in placenta, placental membranes and peritoneum of patients with hypertensive disorder complicating pregnancy. Zhonghua Fu Chan Ke Za Zhi 43: 497-501, 2008 (In Chinese).

8. Salmani D, Purushothaman S, Somashekara SC, et al: Study of structural changes in placenta in pregnancy-induced hypertension. J Nat Sci Biol Med 5: 352-355, 2014.

9. Okawara M, Seki H, Matsuoka K, et al: Examination of the expression of cyclooxygenase-2 in placenta villi from sufferers of pregnancy induced hypertension. Biol Pharm Bull 32: 2053-2056, 2009.

10. Henao-Mejia J, Williams A, Goff LA, et al: The microRNA miR-181 is a critical cellular metabolic rheostat essential for NKT cell ontogenesis and lymphocyte development and homeostasis. Immunity 38: 984-997, 2013.

11. Zhu Y, Wu J, Li S, et al: The function role of miR-181a in chemosensitivity to adriamycin by targeting $\mathrm{Bcl}-2$ in low-invasive breast cancer cells. Cell Physiol Biochem 32: 1225-1237, 2013.

12. Zhai XF, Fang FF, Liu Q, et al: MiR-181a contributes to bufalin-induced apoptosis in PC-3 prostate cancer cells. BMC Complement Altern Med 13: 325, 2013.

13. Wang XF, Shi ZM, Wang XR, et al: MiR-181d acts as a tumor suppressor in glioma by targeting K-ras and Bcl-2. J Cancer Res Clin Oncol 138: 573-584, 2012.

14. Kronski E, Fiori ME, Barbieri O, et al: miR181b is induced by the chemopreventive polyphenol curcumin and inhibits breast cancer metastasis via down-regulation of the inflammatory cytokines CXCL1 and -2. Mol Oncol 8: 581-595, 2014.

15. Yan JJ, Zhu XM, Yin GW, et al: Effect of human miR-181a on function of trophoblast cell line HTR-8/SVneo. Zhonghua Lin Chuang Yi Shi Za Zhi (Dian Zi Ban) 7: 136-138, 2013 (In Chinese).

16. Simone TM and Higgins PJ: Low molecular weight antagonists of plasminogen activator inhibitor-1: therapeutic potential in cardiovascular disease. Mol Med Ther 1: 101, 2012.

17. Yang Z, Wan X, Gu Z, et al: Evolution of the mir-181 microRNA family. Comput Biol Med 52: 82-87, 2014.

18. Madonna R and De Caterina R: Cellular and molecular mechanisms of vascular injury in diabetes - part I: pathways of vascular disease in diabetes. Vascul Pharmacol 54: 68-74, 2011.

19. R Cawyer C, Horvat D, Leonard D, et al: Hyperglycemia impairs cytotrophoblast function via stress signaling. Am J Obstet Gynecol: May 1, 2014 (Epub ahead of print).

20. Su L, Liu R, Cheng W, et al: Expression patterns of microRNAs in porcine endometrium and their potential roles in embryo implantation and placentation. PLoS One 9: e87867, 2014.

21. Estella C, Herrer I, Moreno-Moya JM, et al: miRNA signature and Dicer requirement during human endometrial stromal decidualization in vitro. PLoS One 7: e41080, 2012. 